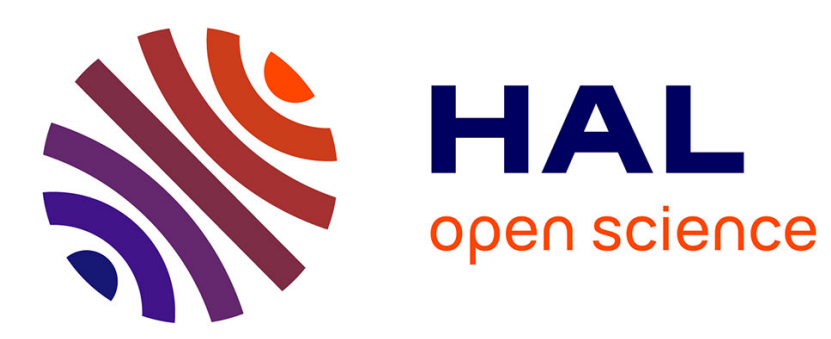

\title{
Structure de bandes expérimentale de TaS2-1 T déterminée par photoémission angulaire
}

\author{
R. Mamy, P. Thiry, G. Vachier, A. Couget
}

\section{To cite this version:}

R. Mamy, P. Thiry, G. Vachier, A. Couget. Structure de bandes expérimentale de TaS2-1 T déterminée par photoémission angulaire. Journal de Physique Lettres, 1981, 42 (4), pp.79-82. 10.1051/jphyslet:0198100420407900 . jpa-00231878

\section{HAL Id: jpa-00231878 https://hal.science/jpa-00231878}

Submitted on 1 Jan 1981

HAL is a multi-disciplinary open access archive for the deposit and dissemination of scientific research documents, whether they are published or not. The documents may come from teaching and research institutions in France or abroad, or from public or private research centers.
L'archive ouverte pluridisciplinaire HAL, est destinée au dépôt et à la diffusion de documents scientifiques de niveau recherche, publiés ou non, émanant des établissements d'enseignement et de recherche français ou étrangers, des laboratoires publics ou privés. 


\title{
Structure de bandes expérimentale de $\mathrm{TaS}_{2}-1 \mathrm{~T}$ déterminée par photoémission angulaire
}

\author{
R. Mamy $(*)$, P. Thiry $(* *)$, G. Vachier $(* * *)$ et A. Couget $(*)$ \\ $\left(^{*}\right)$ Laboratoire de Physique des Solides $\left({ }^{+}\right)$, Université Paul-Sabatier, 31062 Toulouse Cedex, France \\ (Reçu le 29 octobre 1980, accepté le 5 janvier 1981)
}

Résumé. - La structure de bandes de valence de $\mathrm{TaS}_{2}-1 \mathrm{~T}$ a été déterminée expérimentalement, à la température ambiante, le long de la ligne $\Gamma \mathrm{KMK}$ de la zone de Brillouin à l'aide de photons de $40 \mathrm{eV}$. On trouve que la bande $\mathrm{d}_{z^{2}}$ est au-dessous du niveau de Fermi et on explique ce résultat par l'effet d'ondes de densité de charge.

\begin{abstract}
Valence energy bands of $1 \mathrm{~T}-\mathrm{TaS}_{2}$ were experimentally determined, at room temperature, along the $\Gamma \mathrm{KMK}$ line in the Brillouin zone using $40 \mathrm{eV}$ photons. The $\mathrm{d}_{z^{2}}$ band is found to be below the Fermi level and this result is explained by the effect of charge density waves.
\end{abstract}

1. Introduction. - $\mathrm{TaS}_{2}-1 \mathrm{~T}$ est un matériau lamellaire hexagonal dont les figures $1 a$ et $b$ rappellent la zone de Brillouin avec ses points de haute symétrie et une coupe de l'espace réciproque. Il est métallique à la température ambiante et l'existence d'ondes de densité de charge y a été clairement démontrée [1].

Pour de tels cristaux lamellaires, les mesures de photoémission angulaire permettent de déterminer facilement la dispersion des bandes de valence [2]. En effet, l'hypothèse de la conservation de la compo-

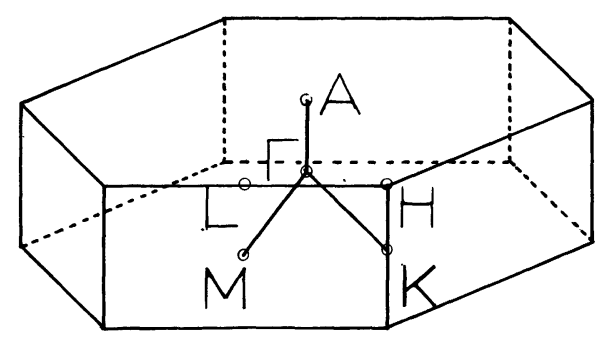

a) sante tangentielle du vecteur d'onde électronique $\mathbf{k}$ à la traversée de la surface de l'échantillon donne :

$$
k_{\|}=\left(2 m E_{\mathrm{c}} / \hbar^{2}\right)^{1 / 2} \sin \theta
$$

où $E_{\mathrm{c}}=\hbar \omega-W+E_{\mathrm{i}}$, $\hbar \omega$ étant l'énergie des photons,

$E_{\mathrm{c}}$ l'énergie cinétique et $W$ le travail de sortie de l'électron,

$E_{\mathrm{i}}$ l'énergie de l'état initial et $\theta$ l'angle d'émergence.

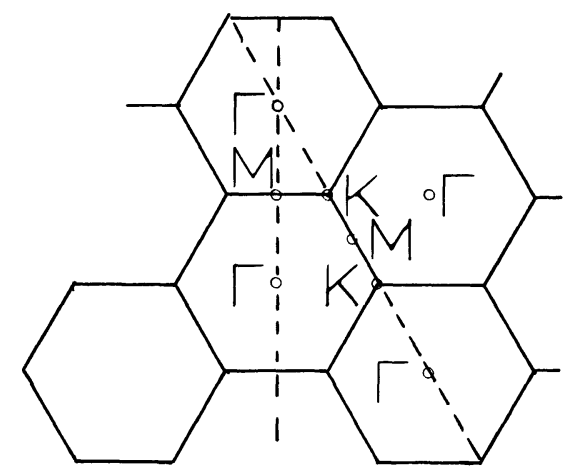

b)

Fig. 1. - (a) Zone de Brillouin et (b) plan 001 de l'espace réciproque de $\mathrm{TaS}_{2}-1 \mathrm{~T}$.

[(a) Brillouin zone and $(b) 001$ plane in reciprocal space of $\left.1 \mathrm{~T}-\mathrm{TaS}_{2}.\right]$

$\left({ }^{+}\right)$Associé au C.N.R.S.

(**) Laboratoire d'Utilisation du Rayonnement Electromagnétique (L.U.R.E.), Université de Paris-Sud, 91405 Orsay, France.

$\left.{ }^{* * *}\right)$ Laboratoire de Chimie des Matériaux, Université de

Provence, Centre Saint-Charles, 13331 Marseille Cedex 3, France. 
On peut ainsi associer à chaque énergie $E_{\mathrm{i}}$ un vecteur d'onde $k_{\|\|}$, et par suite de la faible dispersion des bandes le long de la direction perpendiculaire aux feuillets, on obtient une bonne approximation de la dispersion parallèlement à ceux-ci.

2. Appareillage. - Les mesures ont été faites à la température ambiante dans l'enceinte de photoémission angulaire installée au L.U.R.E. $\left({ }^{* *}\right)$ à Orsay [3]. Dans cette enceinte, la qualité de la surface de l'échantillon peut être contrôlée par spectrométrie Auger et par diffraction d'électrons lents. Le porteéchantillon peut tourner autour d'un axe vertical pour régler l'incidence des photons, et d'un axe horizontal perpendiculaire à la surface de l'échantillon pour régler l'angle $\psi$ d'émergence des photoélectrons (Fig. 2). La rotation de l'analyseur d'électrons autour du même axe vertical que le porteéchantillon permet le réglage de l'angle $\theta$ d'émergence (Fig. 2).

L'échantillon clivé sous ultra-vide $\left(\sim 5 \times 10^{-10}\right.$ torr $)$ est ensuite orienté par diffraction d'électrons lents.

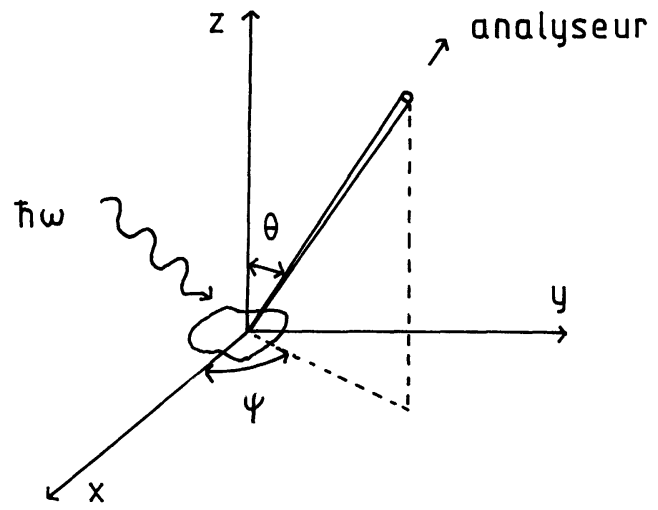

Fig. 2. - Angles $\theta$ et $\psi$ d'analyse des photoélectrons. [ $\theta$ and $\psi$ angles of photoelectron analysis.]

3. Résultats. - Nous avons choisi de faire les mesures avec des photons d'énergie fixée $\hbar \omega=40 \mathrm{eV}$ en polarisation $\mathrm{p}$ et un angle $\psi$ tel que l'on explore la direction $\Gamma \mathrm{KMK}$ de l'espace réciproque en faisant varier l'angle $\theta$ de 0 à $60^{\circ}$. Nos mesures complètent donc celles qui ont été faites le long de $\Gamma \mathrm{M}$ [4].
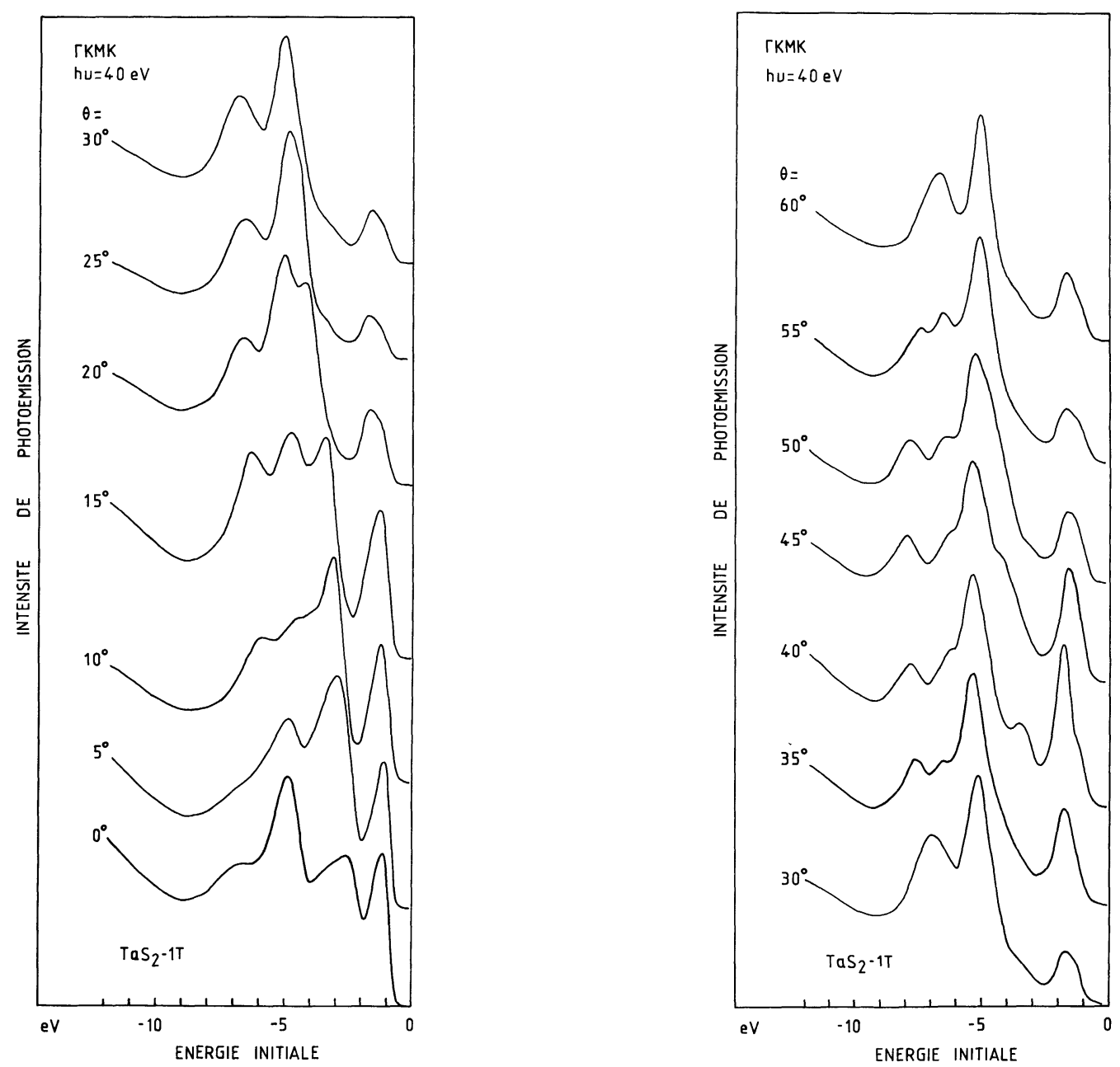

Fig. 3. - Courbes de distribution énergétique des photoélectrons en fonction de l'angle $\theta$. 
La figure 3 montre les courbes de distribution énergétique (C.D.E.) ainsi obtenues. Nous avons porté sur le diagramme de la figure 4 les principales structures de ces courbes en associant à chacune son vecteur d'onde $k_{\|}$et son énergie $E_{\mathrm{i}}$. Pour tracer les bandes expérimentales, nous avons respecté la symétrie par rapport à $\mathbf{M}$; le léger écart entre les points expérimentaux et les courbes ainsi tracées doit être attribué à la dispersion le long de $k_{z}$ et à l'imprécision sur le réglage en $\psi$.

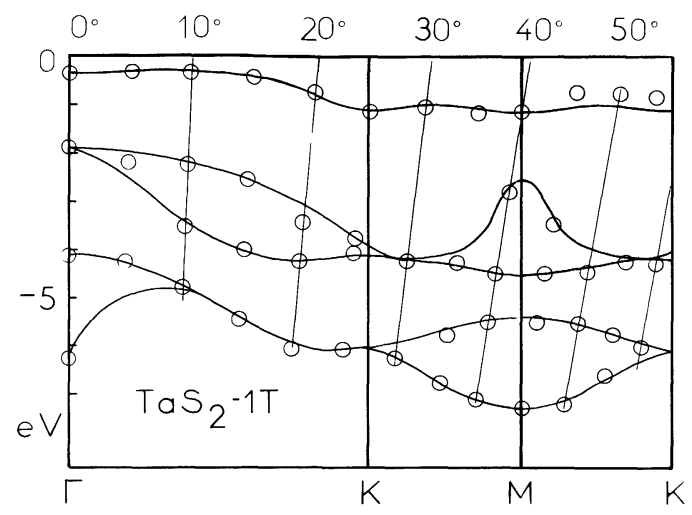

Fig. 4. - Dispersion selon $\Gamma \mathrm{KMK}$ des structures des C.D.E. donnant les bandes de valence expérimentales.

[Dispersion along $\Gamma \mathrm{KMK}$ of the C.D.E. structures giving experimental valence bands.]

La figure 5 présente les bandes de valence de $\mathrm{TaS}_{2}-1 \mathrm{~T}$ calculées par L. F. Mattheiss [5] : la partie occupée de la bande $d_{z^{2}}$ apparaît autour du point $M$; au-dessous se trouvent les six bandes dues principalement aux états $p$ du soufre. Les points expérimentaux de la figure 4 y sont reportés en faisant coïncider au point $M$ mesures et calculs pour la bande $d_{z^{2}}$. On constate ainsi un très bon accord de l'expérience avec la théorie pour les quatre bandes $\mathrm{p}$ observées. Par contre, la bande $d_{z^{2}}$ expérimentale est bien visible tout le long de la ligne, ne passant donc jamais au-

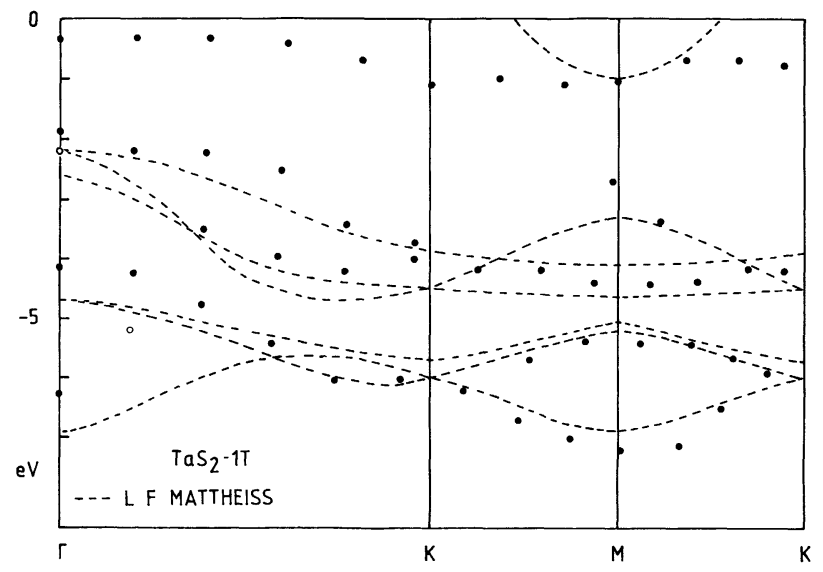

Fig. 5. - Comparaison des résultats expérimentaux et de ceux des calculs de L. F. Mattheiss.

[Comparison of experimental and of L. F. Mattheiss computational results.] dessus du niveau de Fermi, contrairement aux prévisions [5, 6]. Ce résultat est en accord avec celui des mesures de réflectivité infra-rouge [7] montrant l'absence de comportement métallique de $\mathrm{TaS}_{2}-1 \mathrm{~T}$ à basse température.

4. Discussion. - Nous pensons que ces comportements anormaux proviennent de la modification de la surface de Fermi par la déformation périodique du réseau : aux températures inférieures à $190 \mathrm{~K}$, l'existence de vecteurs d'onde critiques et aussi une forte interaction électron-phonon $[1,9]$ entraînent l'apparition d'un super-réseau commensurable (rapport $\sqrt{13} \times \sqrt{13})$ et de l'onde de densité de charge associée, et, par suite, une zone de Brillouin réduite et l'ouverture de gaps au niveau de Fermi par ailleurs responsables du changement de signe du coefficient de Hall [8].

La photoémission à partir d'états situés sous le niveau de Fermi et non prévus par les calculs de bandes peut s'expliquer de diverses manières. Nous négligerons l'effet de transitions non-directes et d'états de surface et retiendrons surtout l'effet des ondes de densité de charge.

La bande $\mathrm{d}_{z^{2}}$ de $\mathrm{TaSe}_{2}-2 \mathrm{H}$ [10] a été recalculée en tenant compte de cet effet. En même temps que la formation de gaps, on constate un abaissement des bandes proches du niveau de Fermi ; c'est ce dernier phénomène qui est visible dans nos résultats sur $\mathrm{TaS}_{2}-1 \mathrm{~T}$.

Nous pensons que l'abaissement de la bande $d_{z^{2}}$ doit être plus prononcé dans le cas de $\mathrm{TaS}_{2}-1 \mathrm{~T}$ que dans celui de $\mathrm{TaSe}_{2}-2 \mathrm{H}$ pour les raisons suivantes :

L'amplitude de la déformation périodique du réseau est plus grande dans le premier cas comme l'indiquent les résultats de diffraction d'électrons, de neutrons, ou de rayons $\mathrm{X}[1,9]$.

Les résultats de mesures de photoémission $X$ [13] suggèrent une forte modulation de la densité de charge autour de l'ion Ta (de l'ordre de 1 électron) entrâ̂nant un éclatement d'environ $0,6 \mathrm{eV}$ de chacun des états $4 \mathrm{f} \mathrm{du} \mathrm{Ta}$, cet éclatement n'est pas détectable dans le cas du polytype $2 \mathrm{H}$.

Les mesures de réflectivité infra-rouge [7] mettent en évidence, pour $\mathrm{TaS}_{2}-1 \mathrm{~T}$ seulement, la disparition du comportement métallique. Il faut remarquer que nos mesures de photoémission ont été faites à température ambiante à laquelle le super-réseau est incommensurable et le composé métallique, alors que nous mettons en évidence un comportement plutôt caractéristique de la phase basse température. Ceci peut s'expliquer par l'existence de domaines commensurables séparés par des défauts de phase [11] (qui disparaissent au-dessous de $190 \mathrm{~K}$ ). L'abaissement de la bande $d_{z^{2}}$, caractéristique de la phase commensurable, est mis en évidence, à la température ambiante, par les transitions optiques surtout sensibles à l'ordre à courte distance [12]. 
Nous pensons que ce comportement de la bande $d_{z^{2}}$ dans la phase commensurable pourrait expliquer les propriétés particulières de $\mathrm{TaS}_{2}-1 \mathrm{~T}$ évoquées cidessus.
Remerciements. - Nous tenons à remercier Y. Pétroff, J. Lecante et leur équipe pour l'aide qu'ils ont apportée à ce travail, ainsi que le personnel du Laboratoire de l'Accélérateur Linéaire et en particulier le groupe « Anneaux ».

\section{Bibliographie}

[1] Wilson, J. A., Di Salvo, F. J., Mahajan, S., Adv. Phys. 24 (1975) 117.

[2] Hughes, H. P., Liang, W. Y., J. Phys. C 6 (1973) 1684.

[3] LeCANTE, J., Le Vide 187 (1977).

[4] Smith, N. V., Traum, M. M., Phys. Rev. B 11, 6 (1975) 2087.

[5] Mattheiss, L. F., Phys. Rev. B 8, 8 (1973) 3719.

[6] Wooley, A. M., WeXler, G., J. Phys. C 10 (1977) 2601.

[7] Barker, A. S., Ditzenberger, J. A., Di Salvo, F. J., Phys. Rev. B 12, 6 (1975) 2049.
[8] Inada, R., Onuki, Y., Tanuma, S., Physica 99B (1980) 188. [9] Friend, R. H., Jérome, D., J. Phys. C 12 (1979) 1441.

[10] Campagnoli, G., Gustinetti, A., Stella, A., Tosatti, E., Phys. Rev. Lett. 38, 2 (1977) 95.

[11] Fazekas, P., Tosatti, E., Physica 99B (1980) 183.

[12] Tosatti, E., Communication privée.

[13] Hughes, H. P., Pollak, R. A., Philos. Mag. 34, 6 (1976) 1025. 